\title{
Modeling, Simulation and Control of an Aircraft with Morphing Wing
}

\author{
Leonardo Barros da Luz*, Wilcker Neuwald Schinestzki ${ }^{*}$, Carlos Eduardo de Souza**, and Pedro Paglione ${ }^{\dagger}$ \\ *Aerospace Engineering Graduation Course, Federal University of Santa Maria, Santa Maria, Brazil \\ ${ }^{* * *}$ Mechanical Engineering Department, Federal University of Santa Maria, Santa Maria, Brazil \\ $\dagger$ Visiting Professor, Federal University of Santa Maria, Santa Maria, Brazil \\ ${ }^{1}$ E-mail/leobdl.formula@gmail.com,wilcker.formula@gmail.com, carlos.souza@ufsm.br,pedro.paglione@gmail.com
}

\begin{abstract}
The technological advances, mainly in the development of new materials, recovered the interest in the application of morphing wings in aircraft. Due to the potential of replacing conventional control surfaces by morphing surfaces, the present work presents the modeling of aerodynamics, dynamics and control design of an aircraft with morphing wings. The morphing concept is given by changing the camber of the trailing edge along the wingspan. For aerodynamics modeling, it was adopted unsteady strip theory and, for dynamics modeling, it was used rigid body mechanics, considering the displacement of the center of mass and the time-varying inertia tensor. Finally, control design is performed using Exponential Mapping Controller (EMC) method. The results showed that, for the adopted variable geometry configuration, the influence of the center of mass displacement and the inertia variation on the aircraft behavior were insignificant, whereas the influences of the unsteady aerodynamics were significant. Consideration of the unsteady aerodynamic effects increases the magnitude of the aircraft movements, necessitating a greater control action.
\end{abstract}

Keywords: Morphing wings, Modeling, Aerodynamic, Dynamics, Control

\section{Introduction}

The development of aircraft over the years led to its use in a variety of applications. The research about flight performance improvement is inserted in this perspective, due to the spread in vehicular electronic computation capacity, what allows, today, for fast computations at a very low cost, and consequently, use in any kind of aircraft.

One of the alternatives for performance improvement relies on the development of more efficient aerodynamic flight controls, specially the variable geometry mechanisms concept. Those are the mechanisms that are designed to adapt itself to changes in the mission environment [1]. The development of such mechanisms seeks inspiration from nature, specially in the birds flight, which achieved a very efficient condition because of biological evolution [2].

The inspiration in nature was already present in the early years of aviation development. In the XIX century, many visionaries developed bird inspired mechanisms that allowed geometry changes aiming performance improvement or flight control [3]. However, the demand for aircraft made of stiffer materials in the place of flexible ones, prevented their further development, and those mechanisms could not be found anymore in most aircraft [2].

Nowadays, the use of variable geometry mechanisms is possible again because of technological development in differ- ent fields, specially in the materials and structures area, what brought the possibility of designing flexible yet fail safe structures. The work of [4] described many aircraft designs that consider variable geometry concepts. On example is the F-14 fighter, which alters its sweep in flight to achieve better performance on different flight phases. The more recent work of [5] describes the development of an aircraft that has all its geometry variable using an innovating structural concept.

\subsection{The variable geometry devices}

The variable geometry devices can be divided into two categories: discrete and continuous [2]. The discrete ones are those found in conventional aircraft, which have single functionalities operating in some flight phases, such as the flaps, slats and rectractable landing gears. The continuous ones usually have multiple functionalities, operating in different flight phases. One example is the bird wing.

\subsection{The Categories of Continuous Changes in Wing Geo- metry}

In [4], the different ways of changing wing geometry are divided between changing shape in wing plan, out of wing plane and in profile. Changes in wing plan shape include changes in wingspan, chord and fluff, while out-of-plane shape changes include variations in torsion, dihedral and flexion. For profile changes, the parameters that vary are arching and thickness. 
For the profile change, [6] presents two distinctions for variations in arching, leading edge variation and trailing edge variation.

The work of [7] presents a very interesting concept of a variable geometry wing, which can be characterized in the category of change in trailing edge bending, as [6]. The concept is given the Spanwise Morphing Trailing Edge (SMTE), which consists of changing the incidence of the trailing edge smoothly over the entire wingspan. To make this smooth transition, active and passive surfaces are used, active surfaces are responsible for the trailing edge movement, while passive surfaces transition to the other active surface.

To carry out the studies of this work, we opted for the SMTE concept of [7], because it has a high application potential, directly impacting the autonomy of the aircraft.

\subsection{The Advantages of Using Variable Geometry Wing}

The use of wings with variable geometry can mainly affect the aerodynamics and its control, seeking to increase its performance. In the work of [6] the advantages of each of the variable geometry wing categories are presented. The advantages for different types of variable geometry are listed in the items below.

- Variable camber: It is capable of changing the lift distribution, having advantages in the takeoff and landing phases and can be applied at the trailing or leading edge. Lead edge application can be a lower noise and drag alternative to conventional slats. Application at the trailing edge can reduce drag, making control surfaces more efficient.

- Variable thickness: You can change the drag of the profile by changing its thickness, directly impacting the location of the transition point from laminar to turbulent regime.

- Variable wingspan: Aircraft with high elongation have low maneuverability and high aerodynamic efficiency, however those with low aspect ratios have good maneuverability and low aerodynamic efficiency. The variable wingspan can enjoy the advantages of low aspect ratios and high aspect ratios.

- Variable sweep: Variable sweep can combine the advantages of a non-sweep wing at low speed, take-off and landing stages with the advantages of sweep wings at high speed speeds.

- Variable twist: Variable twisting can relieve maneuvering and bursting loads. In addition, the twisting of the wing may alter the lift distribution along the wingspan and may have the function of a control surface.

In the experimental work of [8] data were obtained that demonstrated an improvement in the aerodynamic efficiency of the aircraft with the use of a variable wingspan, showing a $17 \%$ increase in autonomy. In addition, FlexSys, founded in 2000 by Dr. Sridhar Kota, indicates that using its FlexFoil technology can reduce drag by a range of $5 \%$ to $12 \%$ for long-range fixed wing aircraft, representing a huge fuel economy [9]

\subsection{Modelings}

To fulfill the objectives of the work, the modeling of kinematics and dynamics, aerodynamics and control is required. Therefore, in the subsections below, the literature review of the kinematics and dynamics modeling applied to aircraft with variable geometry in subsection 1.4.1, aerodynamics in subsection 1.4.2 and the control application in subsection 1.4 .3 .

\subsubsection{Kinematics and Dynamics Modeling}

To perform the dynamics modeling of a variable geometry wing aircraft, one can choose two different techniques, adopt the aircraft with a single rigid body or as the union of several rigid bodies.

In the works of [10] and [11] are presented modeling of the dynamics of a wing aircraft with variable geometry using rigid body mechanics. However, some effects that arise when adopting a change in geometry should be taken into account. The main effect would be a significant change in the center of gravity and moments of inertia, so the inertia tensor is a function in time and has a dynamics, which may be close to the frequency ranges of rigid body dynamics.

Due to the considerations provided by [11], it is clear that the equations of standard rigid body motion cannot be applied. An alternative would be to use multibody dynamics methods, but depending on the choice of method one can find large systems of equations, resulting in a significantly higher computational cost. So in [12] another approach to modeling is provided which consists of continuing to treat the aircraft as a single body but utilizing the displaced center of mass of the origin and relaxing the stiffness condition, making the inertia tensor an explicit function in time. This approach may be a more efficient alternative, because its computational cost is lower compared to the dynamics of multiple bodies, and due to these factors, this approach was chosen for this work.

In [13] aircraft dynamics modeling is performed using a tool called SimMechanics, present in Matlab software, which uses the standard Newtonian dynamics of forces and torques. SimMechanics is a block diagram modeling environment where a body can be modeled by joining multiple rigid bodies through joints.

\subsubsection{Aerodynamic Modeling}

Aerodynamic methods applied to wings with variable geometry are divided into two broad categories, stationary and non-stationary. Moreover, within these categories one can have a division between linear and nonlinear methods.

The linear methods, both stationary and non-stationary, are based on potential flow theory, having a restricted application to thin airfoils and small angles of attack. To overcome these constraints of linear methods, nonlinear aerodynamic methods such as Computational Fluid Dynamics (CFD) are used. 
These, however, require a higher computational cost.

In [6] a review of the different aerodynamic methods used in wing modeling with variable geometry was performed. For the finite wing modeling within the stationary method category, the most commonly used are the Vortex Lattice Method (VLM) and the nonlinear vortex lattice method. For the nonstationary method category, the use of Unsteady Vortex Lattice Method (UVLM) and Doublet Lattice Method (DLM) is highlighted. It is also noted the large use of CFD, which may be non-stationary and stationary, the tendency of increasingly using CFD in these studies is due to its ability to obtain results very close to experimental, especially in the nonlinear region. Finally, [6] concludes that the UVLM method has great potential for modeling involving bending, thickness, torsion and wingspan alterations.

In the aerodynamic modeling performed by [14], we highlight the use of CFD with a Spalart-Allmaras turbulence model, a one-equation model, which has a lower computational cost than other models. turbulence. In [15], the turbulence model used was $k$ - omega, a two equation model, which requires a higher computational cost than Spalart-Allmaras, but the results obtained were very close to those obtained. experimental results.

In the work of [16] the aerodynamic method used is strip theory, with the generalized non-stationary theory of Theodorsen applied to an airfoil. The results for the theory's predicted flutter velocity and frequency were very close to the experimental ones.

In the work of [17] a comparative study is carried out, evaluating loads in flight, between quasi-stationary band theory, Vortex Lattice Method (VLM), non-stationary band theory and Doublet Lattice Method (DLM). The author concludes that non-stationary band theory is the best candidate for use in initial analysis, considering the relationship between computational cost and correct prediction of aerodynamic effects. So, in this work, it was decided to use the non-stationary band theory, with modifications to include inflection, dihedral and profile characteristics.

\subsubsection{Control Application}

There are different control techniques that can be applied to control aircraft with variable geometry, but some complications arise due to the dependence of dynamics on changing geometry. There are two ways to handle control of aircraft with variable geometry, as suggested by [11]. Variable geometry can be considered as a configuration change requiring different controllers in each configuration, or variable geometry can be studied as the control method. Using variable geometry as control effectors, problems of non-unique solutions arise as the number of control variables. Due to the problem of non-unique solutions, the optimal control allocation method may be an alternative.

In [11] some complications are presented in the control design phase of a variable geometry wing aircraft, as in one of the primordial stages of the control design, the system linearization. In the linearization step a linear system of a dynamics described as

$$
\dot{x}=f(x, u)
$$

However, for an aircraft with variable geometry, dynamics also become a function that depends on the $\mu$ geometry change setting and the $\dot{\mu}$ surface rate of change. Therefore, the dynamics should be described as:

$$
\dot{x}=f(x, u, \mu, \dot{\mu})
$$

In the work of [6] a review of the control techniques used in aircraft with variable geometry is presented, highlighting the use of the pseudo-inverse allocation method and quadratic programming allocation within the actuator constraints. In [10] an optimal control technique is used for different flight conditions, with small performance losses in off-project conditions.

For this work, we opted for the Exponential Mapping Controller (EMC) method, developed by [18]. This method is based on the Sliding Mode Control (SMC) and Neuro-Fuzzy Control (NFN) methods, combining some advantages of both methods, demonstrating an excellent ability to solve dynamic control problems with terms. variations in time with some ease.

\section{Mathematical models}

This section presents the description of the main mathematical models used in the proposed framework. The first model presented in subsection 2.1 is the aircraft's kinematics and dynamics. Modeling the complete dynamics of the aircraft requires the aerodynamic forces, which are provided by the aerodynamic model presented in subsection 2.2. Finally, the control design model is presented in subsection 2.3, which will be responsible for commanding and stabilizing the aircraft.

\subsection{Kinematics and Dynamics Model}

The aircraft motion is represented by the translation and rotation of the body reference system (BRS) relative to the inertial reference system (IRS). The BRS, illustrated in Fig. 2.1. is defined with the axis $X_{b}$ poiting in the direction of the aircraft's nose, axis $Y_{b}$ on the right side of the aicraft, when looking in the positive direction of axis $X_{b}$, and axis $Z_{b}$ points downward. Its origin is displaced from the center of mass of the aircraft. The IRS is fixed at the initial position of the aircraft and at the coincides with the BRS at $t=0$.

The translation is characterized by the position vectors $\mathbf{R}_{0}$, $\mathbf{R}_{\mathrm{cm}}$ and $\mathbf{r}_{\mathrm{cm}}$, whereas the rotation is by the attitude of the BRS towards the IRS. The attitude is obtained using the angles of Euler $\phi, \theta, \psi$ defined with the rotation along the $\mathrm{x}, \mathrm{y}$ and $\mathrm{z}$ axis, respectively. The attitude matrix is given by the rotation sequence $z-y-x$.

The forces and moments acting on the reference systems are defined according to fig. 2, with the positive moments and 


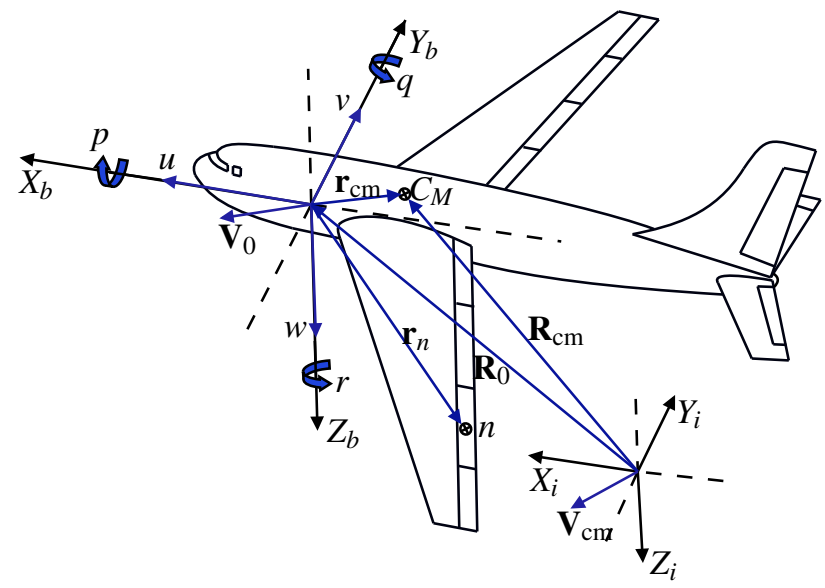

Figure 1: Definition of inertial reference system and body.

body forces in the direction of the BRS axes and the positive aerodynamic forces in the opposite direction of the SAR's axes.

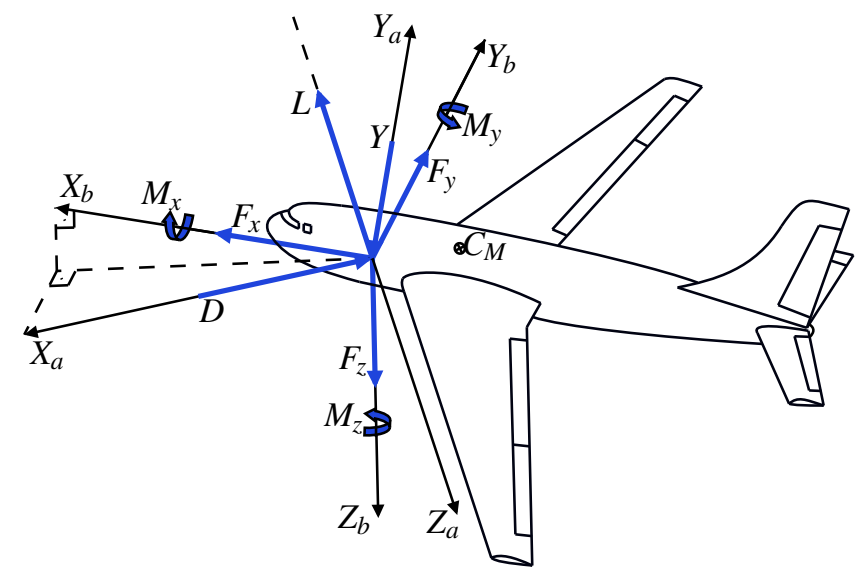

Figure 2: Representation of forces and moments acting on reference systems.

\subsubsection{Dynamics}

The translation dynamics considers the external forces acting on the body according to fig. 2 and the velocities $u, v$ and $w$ according to fig. 2.1. Furthermore, write the velocity $\mathbf{V}_{\mathrm{cm}}$ of the center of mass, illustrated in fig. 2.1, with respect to IRS as:

$$
\mathbf{V}_{\mathrm{cm}}=\mathbf{V}_{0}+\dot{\mathbf{r}}_{\mathrm{cm}}+\omega \times \mathbf{r}_{\mathrm{cm}}
$$

where $\mathbf{V}_{0}$ is the inertial velocity of the BRS origin with components $u, v$ and $w, \omega$ the angular velocity vector of the body with components $p, q$ and $r$, respectively, $\mathbf{r}_{\mathrm{cm}}$ the center of mass position vector relative to the origin of the BRS.

Using Newton's second law, assuming the aircraft has constant mass, then the resulting external force must be equal to the product of the total mass of the aircraft, $m$, by the timederived center of mass velocity, $\dot{\mathbf{V}}_{\mathrm{cm}}$, so that:

$$
\mathbf{F}_{\mathrm{ext}}=m \dot{\mathbf{V}}_{\mathrm{cm}}
$$

By deriving the eq. (3) in time, we obtain, after handle the terms, the translation dynamics for the origin of the BRS, in relation to the inertial system, written as:

$$
\begin{array}{r}
m \dot{\mathbf{V}}_{0}+m\left(\omega \times \mathbf{V}_{0}\right)+m \ddot{\mathbf{r}}_{\mathrm{cm}}+2 m\left(\omega \times \dot{\mathbf{r}}_{\mathrm{cm}}\right) \\
+m \dot{\omega} \times \mathbf{r}_{\mathrm{cm}}+m\left(\omega \times \omega \times \mathbf{r}_{\mathrm{cm}}\right)=\mathbf{F}_{\mathrm{ext}}
\end{array}
$$

where $\mathbf{F}_{\text {ext }}$ is the vector of external forces with components $F_{x}, F_{y}$ and $F_{z}$, respectively, and $m$ is the total mass of the aircraft.

For the rotation dynamics, the rate of change of the amount of total external angular motion is obtained as presented by [12],

$$
\mathbf{M}_{\mathrm{ext}}=\dot{\mathbf{h}}+m\left(\mathbf{r}_{\mathrm{cm}} \times \dot{\mathbf{V}}_{0}\right)
$$

where $\mathbf{M}_{\text {ext }}$ is the total external moment vector with components $M_{x}, M_{y}$ and $M_{z}$, respectively, and $\mathbf{h}$ is the angular momentum BRS, which is expressed as:

$$
\mathbf{h}=\overline{\mathbf{J}} \cdot \boldsymbol{\omega}+\sum_{i=1}^{N_{n}}\left(m_{n}\right)_{i}\left(\left(\mathbf{r}_{n}\right)_{i} \times\left(\mathbf{v}_{n}\right)_{i}\right)
$$

In the above equation, $\overline{\mathbf{J}}$ is the aircraft's inertia matrix, $\mathbf{r}_{n}$ the mass element position vector $n$ shown in fig. 2.1, $\mathbf{v}_{n}$ the velocity vector of the mass element, $m_{n}$ the mass of the mass element, and $N_{n}$ is the amount of mass elements.

Solving the derivative of eq. (7), substituting the result in eq. (6) gives the equation of rotation dynamics described as

$$
\begin{array}{r}
\overline{\mathbf{J}} \cdot \dot{\omega}+\omega \times \overline{\mathbf{J}} \cdot \omega+\dot{\overline{\mathbf{J}}} \cdot \omega+m \mathbf{r}_{\mathrm{cm}} \times\left(\dot{\mathbf{V}}_{0}+\omega \times \mathbf{V}_{0}\right) \\
+\omega \times \sum_{i=1}^{N_{n}}\left(m_{n}\right)_{i} \cdot\left(\mathbf{r}_{n}\right)_{i} \times\left(\mathbf{v}_{n}\right)_{i}+\sum_{i=1}^{N_{n}}\left(m_{n}\right)_{i}\left(\mathbf{r}_{n}\right)_{i} \times\left(\dot{\mathbf{v}}_{n}\right)_{i} \\
+\sum_{i=1}^{N_{n}}\left(m_{n}\right)_{i}\left(\dot{\mathbf{r}}_{n}\right)_{i} \times\left(\mathbf{v}_{n}\right)_{i}=\mathbf{M}_{\mathrm{ext}}
\end{array}
$$

Analyzing the eqs. (5) and (8) we see a dynamic coupling, due to the second derivative of the vector $\mathbf{r}_{\mathrm{cm}}$, giving rise to the term $\dot{\omega}$ in the translation dynamics. This coupling is solved using the approach presented by [19], which consists in solving a coupled linear system, defined as

$$
\left[\begin{array}{cc}
m \overline{\mathbf{I}} & \overline{\mathbf{S}}_{R}^{T} \\
\overline{\mathbf{S}}_{R} & \overline{\mathbf{J}}
\end{array}\right] \cdot\left[\begin{array}{c}
\dot{\mathbf{V}}_{0} \\
\dot{\omega}
\end{array}\right]=\left[\begin{array}{c}
\mathbf{Q}_{F} \\
\mathbf{Q}_{M}
\end{array}\right]
$$

where

$$
\overline{\mathbf{S}}_{R}=m \tilde{\mathbf{r}}_{\mathrm{cm}}
$$




$$
\begin{array}{r}
\mathbf{Q}_{F}=\mathbf{F}_{\mathrm{ext}}-m\left(\omega \times \mathbf{V}_{0}\right)-m \ddot{\mathbf{r}}_{\mathrm{cm}} \\
-2 m\left(\omega \times \dot{\mathbf{r}}_{\mathrm{cm}}\right)-m\left(\omega \times \omega \times \mathbf{r}_{\mathrm{cm}}\right) \\
\mathbf{Q}_{M}=\mathbf{M}_{\mathrm{ext}}-\omega \times \overline{\mathbf{J}} \cdot \omega-\dot{\overline{\mathbf{J}}} \cdot \omega-m \mathbf{r}_{\mathrm{cm}} \times\left(\omega \times \mathbf{V}_{0}\right) \\
-\omega \times \sum_{i=1}^{N_{n}}\left(m_{n}\right)_{i}\left(\mathbf{r}_{n}\right)_{i} \times\left(\mathbf{v}_{n}\right)_{i}-\sum_{i=1}^{N_{n}}\left(m_{n}\right)_{i}\left(\mathbf{r}_{n}\right)_{i} \times\left(\dot{\mathbf{v}}_{n}\right)_{i} \\
-\sum_{i=1}^{N_{n}}\left(m_{n}\right)_{i}\left(\dot{\mathbf{r}}_{n}\right)_{i} \times\left(\mathbf{v}_{n}\right)_{i}
\end{array}
$$

where $\overline{\mathbf{I}}$ the identity matrix, $\overline{\mathbf{S}}_{R}$ the dynamic-coupling matrix, $\tilde{\mathbf{r}}_{\mathrm{cm}}$ the anti-symmetric matrix of $\mathbf{r}_{\mathrm{cm}}, \mathbf{Q}_{F}$ the vector of external forces and $\mathbf{Q}_{M}$ the vector of external moments, considering terms that do not depend on $\dot{\mathbf{V}}_{0}$ and $\dot{\omega}$.

\subsubsection{Kinematics}

For the formulation of the translation kinematics, we consider the position vector $\mathbf{R}_{0}$ in fig. 2.1. with components $x_{0}$ and $y_{0}$ defining the horizontal displacements and $z_{0}$ the altitude considering the origin of IRS at sea level. These components are measured from the BRS origin relative to the IRS origin. Therefore, one can express the inertial velocity $\mathbf{V}_{0}$ as:

$$
\mathbf{V}_{0}=\left[\begin{array}{c}
\dot{x_{0}} \\
\dot{y_{0}} \\
\dot{z_{0}}
\end{array}\right]
$$

Using the velocities $u, v$ and $w$ described in SRC, the translation kinematics can be written as:

$$
\left[\begin{array}{c}
\dot{x_{0}} \\
\dot{y_{0}} \\
\dot{z_{0}}
\end{array}\right]=\left(\overline{\mathbf{C}}_{b}^{i}\right)^{T} \cdot\left[\begin{array}{c}
u \\
v \\
w
\end{array}\right]
$$

To obtain the rotation kinematics, initially the angular velocities in the BRS are described, as

$$
\Omega_{b}=\left[\begin{array}{c}
\dot{\phi}_{b} \\
\dot{\theta}_{b} \\
\dot{\psi}_{b}
\end{array}\right]=\overline{\mathbf{C}}_{1} \cdot \overline{\mathbf{C}}_{2} \cdot\left[\begin{array}{c}
0 \\
0 \\
\dot{\psi}
\end{array}\right]+\overline{\mathbf{C}}_{1} \cdot\left[\begin{array}{c}
0 \\
\dot{\theta} \\
0
\end{array}\right]+\left[\begin{array}{c}
\dot{\phi} \\
0 \\
0
\end{array}\right]
$$

And assume an angular velocity $\omega=[p, q, r]$, in order to write the equation to be solved as:

$$
\Omega_{b}=\left[\begin{array}{c}
p \\
q \\
r
\end{array}\right]
$$

Then, solving the eq. 16 obtains the rotation kinematics described as:

$$
\left[\begin{array}{c}
\dot{\phi} \\
\dot{\theta} \\
\dot{\psi}
\end{array}\right]=\left[\begin{array}{ccc}
1 & \operatorname{sen}(\phi) \tan (\theta) & \cos (\phi) \tan (\theta) \\
0 & \cos (\phi) & -\operatorname{sen}(\phi) \\
0 & \operatorname{sen}(\phi) \sec (\theta) & \cos (\phi) \sec (\theta)
\end{array}\right] \cdot\left[\begin{array}{c}
p \\
q \\
r
\end{array}\right]
$$

\subsubsection{Morphing Modeling}

Aircraft kinematics and dynamics require the first and second derivatives of the position vectors $\mathbf{r}_{\mathrm{cm}}$ and $\mathbf{r}_{\mathrm{n}}$, as well as the first derivative of the $\overline{\mathbf{J}}$ inertia matrix. The complete dynamics of the aircraft is a system of ordinary differential equations, therefore care must be taken with the derivative approximations, because when adopting approximation methods as finite differences a time-step dependent truncation error is entered. This error increases the error in the ordinary differential system solution method.

Therefore, due to the complications introduced by the finite difference approximation, the approximation provided by [12] was used. To obtain the derivatives we use a function or a numerical routine capable of obtaining the parameters of interest as a function of the deflections $\delta$ of each mass element, obtaining the derivatives according to the equations below:

$$
\begin{gathered}
\mathbf{r}_{\mathrm{cm}}=f\left(\delta_{1}, \delta_{2}, . ., \delta_{N n}\right) \\
\dot{\mathbf{r}}_{\mathrm{cm}}=\sum_{k=1}^{N n} \frac{\partial \mathbf{r}_{\mathrm{cm}}}{\partial \delta_{k}} \cdot \dot{\delta}_{k} \\
\ddot{\mathbf{r}}_{\mathrm{cm}}=\dot{\boldsymbol{\delta}}^{T} \cdot\left(\sum_{j=1}^{N n} \sum_{k=1}^{N n} \frac{\partial^{2} \mathbf{r}_{\mathrm{cm}}}{\partial \delta_{j} \partial \delta_{k}}\right) \cdot \dot{\boldsymbol{\delta}}+\sum_{k=1}^{N n} \frac{\partial \mathbf{r}_{\mathrm{cm}}}{\partial \delta_{k}} \cdot \ddot{\delta}_{k}
\end{gathered}
$$

To obtain the derivatives of $\mathbf{r}_{\mathrm{n}}$ and the $\overline{\mathbf{J}}$ inertia matrix just repeat the process performed for the position vector $\mathbf{r}_{\mathrm{cm}}$.

However, using this approximation introduces the dependence with the first and second derivative of the $\delta$ deflections, that is, a second order model of the actuator dynamics is required for each deflection, as follows:

$$
\begin{gathered}
\dot{\delta}_{p}=-2 \zeta \omega_{n} \cdot \delta_{p}+\omega_{n}^{2} \cdot\left(\delta_{c}-\delta\right) \\
\dot{\delta}=\delta_{p}
\end{gathered}
$$

where $\delta_{c}$ is the commanded deflection provided by the control project, and $\delta_{p}$ a variable substitution for the first derivative of $\delta$.

These dynamics must be introduced into the system solution, increasing the number of states of the complete dynamics.

To obtain the values of inertia and center of mass position required for derivative approximations, it is assumed that the panels responsible for the morphing effect are concentrated masses positioned at the center of mass of each panel. Then, the position of the aircraft's center of mass relative to the BRS is obtained by: 


$$
\mathbf{r}_{\mathrm{cm}}=\frac{1}{m} \cdot \sum_{\mathrm{i}=1}^{N_{n}}\left(m_{n}\right)_{i} \cdot\left(\mathbf{r}_{n}\right)_{i}
$$

and the resulting inertia matrix,

$$
\overline{\mathbf{J}}=\overline{\mathbf{J}}_{F}+\sum_{\mathrm{i}=1}^{N_{n}}\left(m_{n}\right)_{i} \cdot\left(\tilde{\mathbf{r}}_{n}\right)_{i} \cdot\left(\tilde{\mathbf{r}}_{n}\right)_{i}^{T}
$$

where $\overline{\mathbf{J}}_{F}$ is the inertia matrix of components that are not part of the morphing structure and $\tilde{\mathbf{r}}_{n}$ is the anti-symmetric matrix of $\mathbf{r}_{n}$.

\subsection{Aerodynamic model}

To obtain the aerodynamic forces and moments, one can make use of stationary, non-stationary or quasi-stationary aerodynamic methods. The difference between these approaches is in considering the effects of flow in time. In the scope of this work, the analysis of the variant effects in time is inserted, therefore the non-stationary approach will be adopted.

In order to understand the adopted method, the topics about the typical subsection on which the method is based, in the subsection 2.2.1, are presented the formulation of the unsteady strip theory, in the subsection 2.2.2, the contribution of the movements of rigid body, in subsection 2.2.3. finally, obtaining the derivatives of stability and control in subsection 2.2 .4

\subsubsection{Typical Section}

For the formulation of unsteady strip theory, two-dimensional formulations are required, such as the analytical equations developed by [20] for a typical section with 3 degrees of freedom, these being the angle $\theta, \delta$ and the displacement h, represented in fig. 3. In order to obtain the complete analytical solution for non-stationary aerodynamic loading, [20] considered a thin profile and two-dimensional, incompressible, potential flow subject to a simple harmonic motion.

Given the modeling assumptions, [20] do perform overlaps potential flows, being divided into a non-circulatory part, related to the airfoil, and another circulating part, related to the vortex mat extending from the trailing edge to infinity. With these potentials, forces and moments can be calculated through the integration of pressures over chord and the relationship between circulatory and non-circulatory forces is given by Theodorsen function $C(k)[21]$.

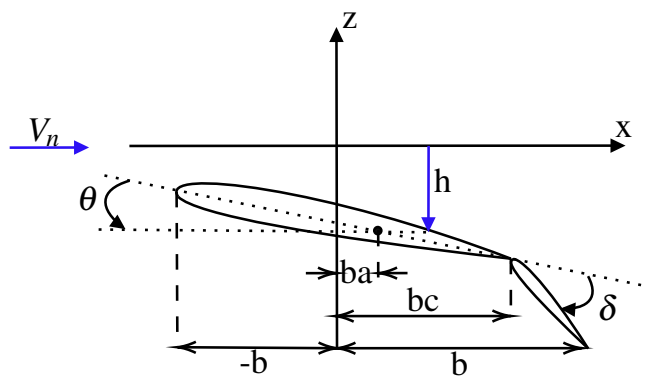

Figure 3: Typical section with three degrees of freedom.
In fig. 3, $V_{n}$ represents the flow velocity, $b c$ the distance between the control surface articulate point and the origin, $b a$ the distance between the elastic axis and the origin, $b$ the semi-chord.

\subsubsection{Unsteady Strip Theory}

Unsteady strip theory is based on the idea of representing three-dimensional aerodynamic flows by dividing the surface of interest into strips along its span, where the solutions developed for a typical section, as illustrated in the previous subsection, apply. Thus, three-dimensional effects such as the wingtip effect are neglected.

For the theory to be able to represent a wing with a sweep and dihedral angle, the modifications presented by [22] must be perform. Furthermore, in the work of [23] the modification is perform to accommodate the characteristics of the profile. Using the formulations provided by both works, the final formulation of the strip theory is called Modified Strip Theory (MST), described by the equations:

$$
\begin{gathered}
Q=\dot{h}+V_{n} \theta+V_{n} \sigma \tan \left(\Lambda_{\mathrm{ea}}\right)+b\left(\frac{C_{l \alpha, n}}{2 \pi}+a c_{n}-a\right) \\
\left(\dot{\theta}+V_{n} \tau \tan \left(\Lambda_{\mathrm{ea}}\right)\right)+\frac{V_{n} \delta}{\pi} T_{10}+\frac{b \dot{\delta}}{2 \pi} T_{11} \\
L=-\pi \rho b^{2}\left[\ddot{h}+V_{n} \dot{\theta}+V_{n} \dot{\sigma} \tan \left(\Lambda_{\mathrm{ea}}\right)-b a\left(\ddot{\theta}+V_{n} \dot{\tau} \tan \left(\Lambda_{\mathrm{ea}}\right)\right)\right] \\
-C_{l \alpha, n} \rho V_{n} b C(k) Q+\pi \rho b^{2} \dot{\delta} T_{4}+\rho b^{3} \ddot{\delta} T_{1} \\
M_{\alpha}=\pi \rho b^{2} V_{n}\left(\dot{h}+V_{n} \sigma \tan \left(\Lambda_{\mathrm{ea}}\right)\right)+\pi \rho b^{3} a\left(\ddot{h}+V_{n} \dot{\sigma} \tan \left(\Lambda_{\mathrm{ea}}\right)\right) \\
-\rho b^{3} V_{n} \dot{\delta}\left(T_{1}-T_{8}-T_{4}(c-a)\right)-\rho b^{4} \ddot{\delta}\left(-T_{7}-T_{1}(c-a)\right) \\
-\pi \rho b^{2}\left(\frac{1}{8}+a^{2}\right)\left(\ddot{\theta}+V_{n} \dot{\tau} \tan \left(\Lambda_{\mathrm{ea}}\right)\right)-\rho b^{2} V_{n}^{2} \delta T_{4} \\
-2 \pi \rho b^{2} V_{n} Q\left(\frac{1}{2}-C(k) \frac{C_{l \alpha, n}}{2 \pi}\left(a-a c_{n}\right)\right) \\
+\pi \rho b^{2} V_{n}^{2}\left(\theta-a b \tau \tan \left(\Lambda_{\mathrm{ea}}\right)\right) \\
C_{l \alpha, n}=\frac{(27)}{\cos \left(\Lambda_{\mathrm{ea}}\right)}, \quad V_{n}=V \cos \left(\Lambda_{\mathrm{ea}}\right), \quad k=\frac{\omega b}{V_{n}}
\end{gathered}
$$

In the above equations $Q$ is the strip downwash, $M_{\alpha}$ the strip pitch moment, $L$ the strip lift, $\sigma$ the strip dihedral, $\tau$ the torsion of the strip, $\Lambda_{\text {ea }}$ the sweep angle relative to the elastic axis, $C_{l_{\alpha, n}}$ angular coefficient of the curve $C_{l} \times \alpha, c$ the dimensionless position of the profile control surface, $a$ the dimensionless position of the elastic profile axis, $a c_{n}$ the dimensionless position of the profile aerodynamic center, and $\rho$ the flow density.

For the discretization of the surface to be in accordance with the formulation presented, the strips must be positioned perpendicularly along the elastic axis. Each of the ranges receives the system of equations presented above. 


\subsubsection{Contribution of Rigid Body Movements}

This subsection presents the contributions of rigid body movements to enable the use of theory in a flight mechanics problem. These contributions are discussed in the work of [24], in order to find the contributions of the aircraft $\alpha$ and $\beta$ angles and the effective angles $\theta_{R}, \sigma_{R}$ and $\Lambda_{R}$ of each strip, as well as the contribution of the angular velocity of the body to the velocity $\dot{h}$ of each strip.

Given a function that relates the effective angles of each strip to the body's $\alpha$ and $\beta$ angles, one can describe the effective angles as:

$$
\theta_{R}=f_{\theta_{R}}(\alpha, \beta), \quad \Lambda_{R}=f_{\Lambda_{R}}(\alpha, \beta), \quad \sigma_{R}=f_{\sigma_{R}}(\alpha, \beta)
$$

Therefore, the angle $\Lambda_{e a}, \theta$ and the displacement $h$ from the formulation of the previous subsection should be replaced, in each strip, with:

$$
\begin{aligned}
\Lambda_{e a} & =\Lambda_{e a}+f_{\Lambda_{R}}(\alpha, \beta) \\
\theta & =f_{\theta_{R}}(\alpha, \beta)-\alpha_{0} \\
h & =y^{\prime} f_{\sigma_{R}}(\alpha, \beta) \cos \left(\Lambda_{e a}\right)
\end{aligned}
$$

where $\alpha_{0}$ is the angle of attack with zero lift of the profile and $y^{\prime}$ is the y coordinate along the elastic axis.

The contribution of the angular velocities $p, q$ and $r$ of the body is understood as an induced velocity with respect to the distance from the elastic axis of the strip to the body system reference point. Then the $\dot{h}, \sigma$ and $\tau$ variables are replaced by:

$$
\begin{aligned}
& \sigma=f_{\sigma_{R}}(\alpha, \beta) \cdot \cos \left(\Lambda_{e a}\right) \\
& \tau=\frac{\partial f_{\theta_{R}}(\alpha, \beta)}{\partial y^{\prime}}-\frac{\alpha_{0}}{\partial y^{\prime}} \\
& \dot{h}=L_{p} p+L_{q} q+L_{r} r+y^{\prime} \cdot \frac{\partial f_{\sigma_{R}}(\alpha, \beta)}{\partial t} \cdot \cos \left(\Lambda_{e a}\right)
\end{aligned}
$$

where $L_{p}, L_{q}$, and $L_{r}$ are the distances between the elastic axis of the strip and the origin of the body system for each angular velocity.

\subsubsection{Stability and Control Derivatives}

The formulation of the stability and control derivatives considers a linearized condition around an equilibrium permanent flight condition, so any state variable $x_{i}$ can be described in relation to its balance plus your disturbance $x_{i}(t)=\left.x_{i}\right|_{\text {eq }}+$ $\Delta x_{i}(t)$. However, the force and moment expressions presented are valid for harmonic motion with frequency $\omega$, so you must express the perturbations in a state variable as $\Delta x_{i}=\Delta \bar{x}_{i} \cdot e^{i \omega t}$.

Writing the expressions of forces and moments in relation to perturbations of state variables $\alpha, \beta, p, q$ and $r$ gives the linearized forces and moments in relation to a movement harmonic. However, the interest is in describing the forces and moments for any movement. For this, the principle is used that any physical response in time can be approximated by a combination of harmonic movements. Therefore, the imaginary variable $i \omega=s$ can be substituted in the formulation and the Theodorsen function $C(k)$ can be used for the frequency domain using the modified 0 and 1 Bessel functions [25].

In the frequency domain we get a state perturbation vector $\Delta \mathbf{x}(s)$, the force $L(s)$, and the momentum $M_{\alpha}(s)$ as:

$$
\Delta \mathbf{x}(s)=\left[\begin{array}{llllll}
\Delta \alpha(s) & \Delta \beta(s) & \Delta p(s) & \Delta q(s) & \Delta r(s) & \Delta \delta(s)
\end{array}\right]^{T}
$$

$$
L(s)=\left.L\right|_{\mathrm{eq}}+\Delta \mathbf{L}(s) \cdot \Delta \mathbf{x}(s)
$$

$$
M_{\alpha}(s)=\left.M_{\alpha}\right|_{\mathrm{eq}}+\Delta \mathbf{M}_{\alpha}(s) \cdot \Delta \mathbf{x}(s)
$$

The expressions of the forces and moments in the eqs. (33) and 34 ) are applied to each range and can be summed vectorically, taking into account the direction of action of each force and moment. By grouping the terms, get a $\overline{\mathbf{A}}_{E}(s)$ matrix for forces and moments in equilibrium and a $\overline{\mathbf{A}}(s)$ matrix for perturbative terms, called the matrix of aerodynamic influence coefficients.

To leave the frequency domain and obtain the temporal response of forces and moments, the inverse Laplace transform must be used. However, the direct application of the transform cannot be performed directly, because Theodorsen $C(s)$ function is not Laplace-invertible. Therefore, one must perform the rational function approximation of the $\overline{\mathbf{A}}(s)$ matrix. The method for approximating the matrix elements used in this work is Roger's method.

With the approximation performed, one can use the inverse Laplace transform and then obtain the forces and moments in the time domain as the expression below.

$$
\mathbf{F}(t)=\left[\begin{array}{llllll}
F_{x}(t) & F_{y}(t) & F_{z}(t) & M_{x}(t) & M_{y}(t) & M_{z}(t)
\end{array}\right]^{T}
$$

$$
\begin{array}{r}
\mathbf{F}(t)=\overline{\mathbf{A}}_{E}(t)+\overline{\mathbf{A}}_{0}(t) \cdot \Delta \mathbf{x}(t)+\left(\frac{b_{r e f}}{V}\right) \overline{\mathbf{A}}_{1}(t) \cdot \Delta \dot{\mathbf{x}}(t)+ \\
\overline{\mathbf{A}}_{2}(t)\left(\frac{b_{r e f}}{V}\right)^{2} \cdot \Delta \ddot{\mathbf{x}}(t)+\sum_{\mathrm{i}=1}^{n_{\mathrm{lag}}} \overline{\mathbf{A}}_{\mathrm{i}+2}(t) \cdot \mathbf{x}_{\mathrm{i}}^{\operatorname{lag}}(t)
\end{array}
$$

where $b_{\text {ref }}$ is the aircraft's reference wingspan, $V$ the total aircraft speed and $\mathbf{x}_{\mathrm{i}}^{\operatorname{lag}}(t)$ o vector of states representing the terms of aerodynamic delay.

Finally, each of the components of the $\mathbf{F}(t)$ vector can be described in terms of the coefficients of influence, called stability derivatives, when referring to state variables, and control, when referring to to control deflections. For dimensionlessness we use the aerodynamic mean chord $c_{r e f}$ and the wing plan area $S_{r e f}$. 


\subsection{Control project model}

This section presents the control design method, with its formulation and characteristics. To understand the method, the necessary steps for the control design in subsection 2.3.1 and the design routine adopted in subsection 2.3.2 are presented.

\subsubsection{The EMC Method}

The EMC method implements an SMC-inspired approach with a heuristically defined nonlinear mapping function. The shape and boundary of the mapping function is defined by the operator. The limit is derived from basic information about the actuators and its shape is defined based on knowledge of system behavior. For EMC implementation, follow these steps:

1. Switched error calculation:

$$
e_{t}=\frac{x_{r e f}-x}{e_{r}}
$$

where $x_{r e f}$ is the reference of any state $x$ and $e_{r}$ the first design parameter.

2. The parameter $e_{t}$ is restricted to being between -1 and 1 :

$$
e_{s}= \begin{cases}-1, & \text { se } e_{t}<-1 \\ e_{t}, & \text { se }-1 \leq e_{t} \leq 1 \\ 1, & \text { se } e_{t}>1\end{cases}
$$

3. Exponential Function Calculation:

$$
u_{e}=\operatorname{sign}\left(e_{s}\right)\left(1-|| e_{s}|-1|^{2^{-B}}\right)
$$

where $\operatorname{sign}\left(e_{s}\right)$ is a function that returns the $e_{s}$ and $B$ sign the second project parameter, usually inserted between 10 and 10.

4. Control action calculation:

$$
u=\frac{u_{\max }-u_{\min }}{2}\left(u_{e}-1\right)+u_{\max }
$$

where $u_{\max }$ and $u_{\min }$ the maximum and minimum limits of the control action.

In practice EMC needs only two parameters and control stops, making it easy to implement and adjust.

\subsubsection{Project Routine}

With the specifications of the steps of the EMC method, you can use it in a design routine. The routine consists of a reference entry and an initial kick of the $e_{r}$ and $B$ parameters for each of the state variables to be traced. In Figure 4 is the system block diagram, with the state variables used in the control project.

The project can be divided into two, controller and stabilizer, as shown above. The controller is responsible for controlling the $V, H$ and $\phi$ tracked variables, while the stabilizer is responsible for stabilizing the aircraft, using as input the comparison between the angles $\theta$ and $\alpha$.

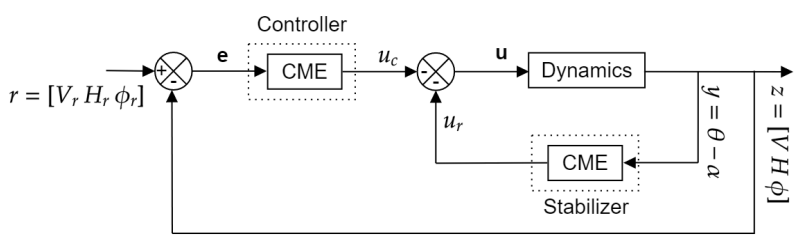

Figure 4: Block diagram for the control project.

The system is resolved with the ode15s function of the MATLAB (C) software, and thereafter the errors are stored between references and tracked states. As a penalty criterion, the time integral multiplied by the absolute value of the error $|e(t)|$ is used, a very useful criterion used to penalize transient responses [26]. The criterion can be described as:

$$
f_{\text {crit }}=\int_{0}^{\infty} t|e(t)| \cdot d t
$$

Finally, the final $e_{r}$ and $B$ parameters are obtained by minimizing the $f_{\text {crit }}$ function, using a minimization function such as fminsearch function of MATLAB (c).

\section{Numerical Studies}

\subsection{Reference Aircraft}

To evaluate the proposed formulation, a simple reference aircraft was chosen, similar to the aircraft presented by [27]. It is a flying wing unmanned aerial vehicle (UAV), seen in Fig. 5. with a reference chord of $0.276 \mathrm{~m}$, wingspan of $1.2 \mathrm{~m}$, total mass of $0.9 \mathrm{~kg}$ and a sweep angle of $30^{\circ}$. In addition, the flight condition is defined as a cruise flight with a speed of $12 \mathrm{~m} / \mathrm{s}$ and an altitude of $100 \mathrm{~m}$.

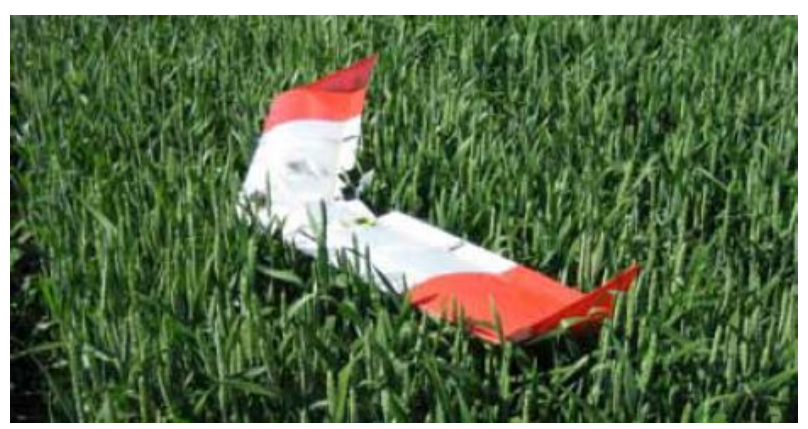

Figure 5: Reference aircraft [27].

However, the mass distribution of this aircraft is unknown. Thus, defining this distribution, becomes a challenge, directly affecting the stability of the aircraft, and making it difficult to integrate the equations system and consequently the control design.

To overcome this problem, a mesh of ten elements along the chord and twenty elements along the span is considered, and each element is considered as a point mass. The aircraft's inertia and center of mass position are computed with equations (23) and 24). Different total masses are defined for each area of the model, so that a mass of $40 \%$ of the total mass is considered to be in the central region, where the propulsive 
system is located, and each half wing has $30 \%$ of total mass. The resulting center of mass is used as reference point for the calculation of moments of inertia. In fig. 6, the elements of each half wing are highlighted in blue, the central region in red, and the center of mass identified with a blue dot. Table 1 shows the values of moments of inertia and the position of the center of mass.

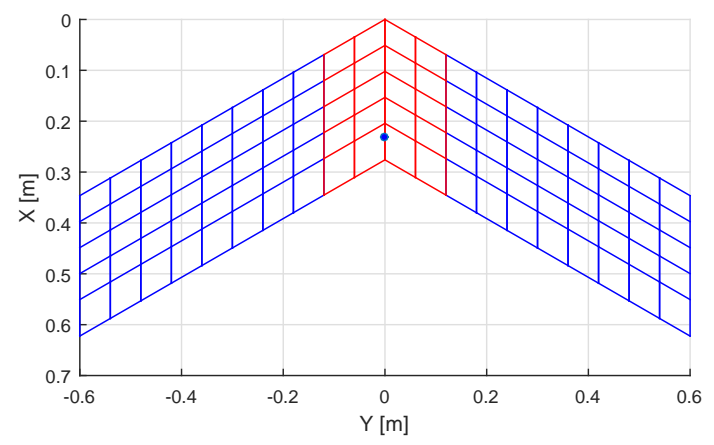

Figure 6: The reference model discretized in elements for mass and inertia computation with different densities.

Table 1: Moment of inertia values and center of mass coordinates of reference aircraft.

\begin{tabular}{lc}
\hline Inertial parameters & Values (S.I) \\
\hline$I_{\mathrm{xx}}$ & 0,0681 \\
$I_{\mathrm{yy}}$ & 0,0116 \\
$I_{\mathrm{zz}}$ & 0,0797 \\
$I_{\mathrm{zx}}=I_{\mathrm{yx}}=I_{\mathrm{zy}}$ & 0 \\
$x_{\mathrm{cm}}$ & 0,2317 \\
$y_{\mathrm{cm}}=z_{\mathrm{cm}}$ & 0 \\
\hline
\end{tabular}

The aerodynamic model is composed of 20 strips, divided into each side, all with the same area, as seen in Fig. 7. There are two control surfaces areas, one at the inner portion of a each half wing, comprising 5 strips, and another at the outer portion, comprising 3 strips. The fuselage area is modeled with a total of 4 strips, which are superposed.

The aircraft has propulsive limitations, with a maximum power of $260 \mathrm{~W}$, so the maximum speed attained by the aircraft is $22.0 \mathrm{~m} / \mathrm{s}$, while the minimum speed is $9.0 \mathrm{~m} / \mathrm{s}$ for no loss of support. In addition, it also has limitations of control surface deflections due to actuator constraints, which are $20^{\circ}$ for maximum deflection and $-20^{\circ}$ for minimum deflection [27].

\subsection{Response to Control Surface Disturbances}

The analysis of the dynamics response to control surface disturbances is important for the understanding of aircraft behavior regarding the use of controls. To perform this analysis, we chose to only disturb the elevator command with a doublet entry of a $\Delta \delta_{p}= \pm 1^{\circ}$ for 2 seconds. In addition, three different dynamics were considered, the first disregards the effects

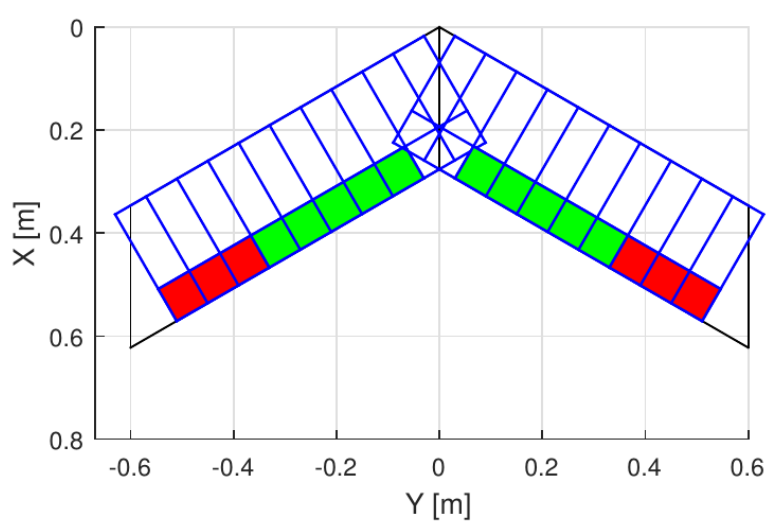

Figure 7: The aerodynamic model, considering the wing discretized into 20 strips.

of non-stationary aerodynamics and temporal variations of inertial parameters, the second disregards only the effects of temporal variations of inertial parameters, and the third only the effects of aerodynamics. non-stationary. In fig. 8 the black results refer to the first dynamics, blue to the second and red to the third when disturbed by the elevator control.

By analyzing the above results it can be seen that the behavior of the aircraft is consistent with the expected modeling, as a positive disturbance in the elevator decreases the angle of attack $\alpha$ and the angle of pitch $\theta$, since This disturbance generates a negative pitching moment. In addition, as the angle of attack decreases, speed increases due to decreased drag, and altitude decreases due to decreased lift. The control result shows the actuator dynamics, which have relatively fast dynamics.

Performing a comparative analysis between the different dynamics, it is clear that the first and third dynamics have identical results, while the second dynamics have different results, but maintains the physical sense. This difference in magnitude of results can be explained by analyzing noncirculatory or apparent mass terms, which consider the displacement of air mass in surface motion, increasing the magnitude of forces and moments acting on motion.

In fig. 9 we find the variations of the inertial parameters when considering the third dynamic, as discussed in the previous paragraph, this dynamic does not influence the results, ie the variations are very small and consequently result in forces. and very small moments.

\section{Conclusions}

The work presented the modeling of a Exponential Mappping Controller applied to morphing wing. The aerodynamic model considers the modified strip theory.

By using the flight dynamics that considers the time-varying displacement of the center of mass and the resulting inertia, it is necessary to use a model able of computing these variables continously and estimate the associate derivatives. Approximations of derivatives of inertial parameters should be performed with caution. The system solution needs a variable 
(a)

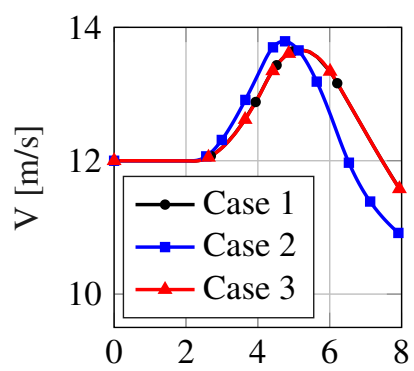

(c)

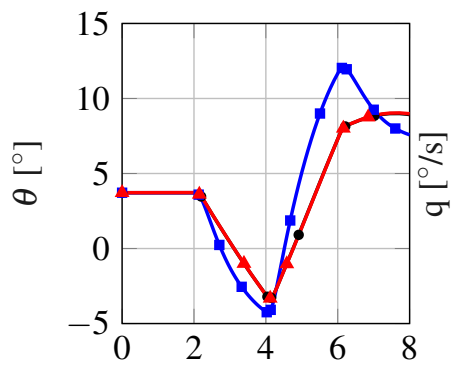

(e)

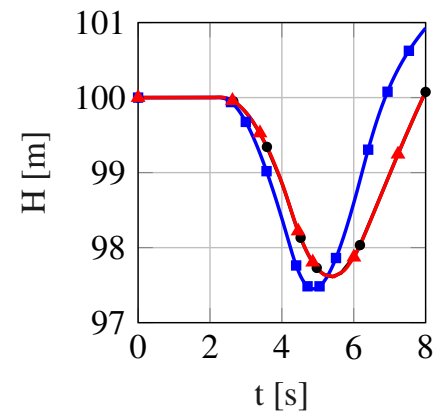

(b)

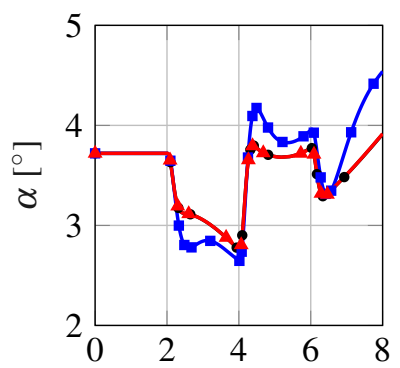

(d)

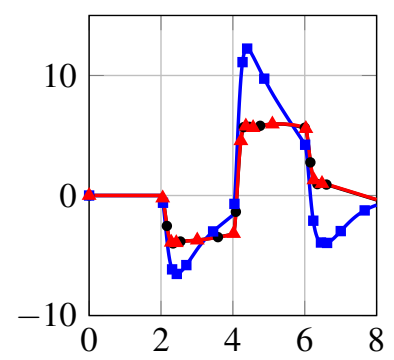

(f)

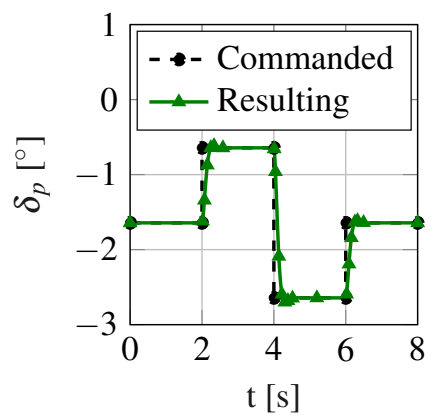

Figure 8: Response of state variables to elevator control disturbance.

time step, which makes it impossible to use more usual derivative approximations, such as the finite difference method. So, to circumvent this problem one can use linear relationships between inertial parameters and deflections, needing to know the dynamics of the actuator.

The aerodynamic method should be chosen carefully, as some non-stationary aerodynamic methods depend on the time step, such as the UVLM, requiring a more complete numerical study when it comes to the union between the equation system solution and the aerodynamics.

For the use of non-stationary strip theory, it is necessary to know the first and second derivatives of state variables, but these quantities are not known before the aerodynamic forces and moments are obtained. To work around this problem, an estimate is made using the linearized dynamics model, considering that the linearized matrices remain constant over time.

The time variations of the center of mass position and the aircraft inertia proved to be insignificant, mainly due to the category adopted for variable geometry. It is possible that a more agressive morphing concept might affect more the flight (a)

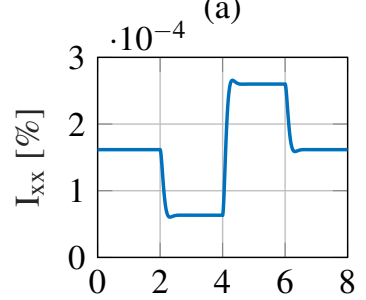

(c)

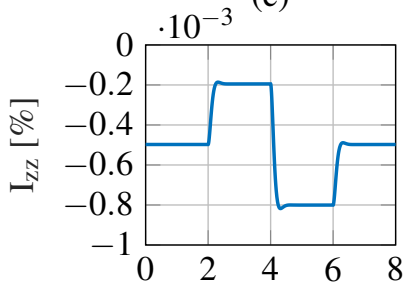

(e)

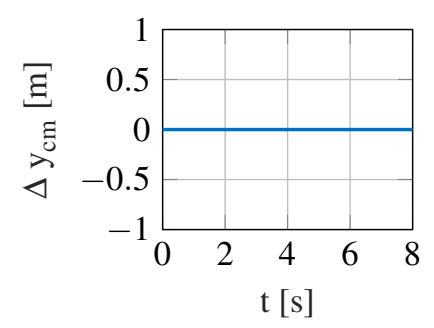

(b)

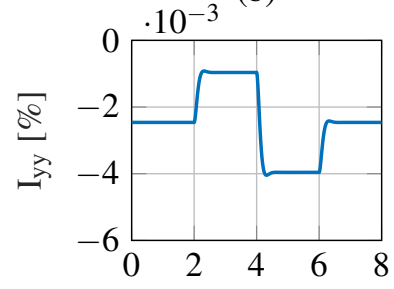

(d)

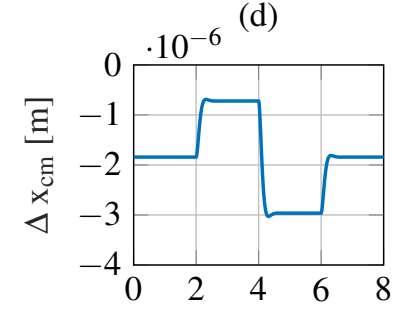

(f)

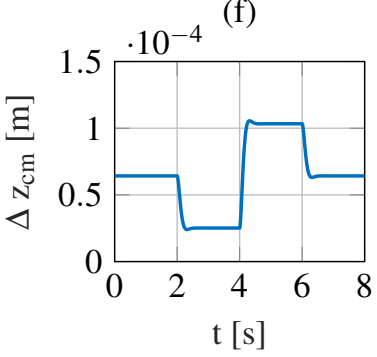

Figure 9: Time variations in center of mass position and moments of inertia for a disturbance in elevator control.

dynamics. This investigation is future step in the present research.

The use of non-stationary aerodynamics is required for a more coherent description of aircraft movement, as non-stationary aerodynamic effects are significant in the aircraft's ultimate behavior.

The control method used was very effective for the simulated cases adopted.

\section{Nomenclature}

\begin{tabular}{lll}
\hline Designation & Denotation & Unit \\
\hline $\mathbf{F}_{\text {ext }}, \mathbf{Q}_{\mathrm{F}}, L$ & Force & $\mathrm{N}$ \\
$\mathbf{M}_{\mathrm{ext}}, \mathbf{Q}_{\mathrm{M}}, M_{\alpha}$ & Moment & $\mathrm{N} . \mathrm{m}$ \\
$\mathbf{h}$ & Angular moment & $\mathrm{N} . \mathrm{rad}$ \\
$\psi, \theta, \phi, \alpha, \beta$ & Angle & $\mathrm{rad}$ \\
$\omega$ & Angular velocity & $\mathrm{rad} / \mathrm{s}$ \\
$\mathbf{V}_{\mathrm{cm}}, \mathbf{V}_{0}, \mathbf{v}_{n}$ & Velocity & $\mathrm{m} / \mathrm{s}$ \\
$\overline{\mathbf{J}}, \overline{\mathbf{J}}_{F}$ & Moment of inertia & $\mathrm{kg} \cdot \mathrm{m}^{2}$ \\
$\mathbf{R}_{\mathrm{cm}}, \mathbf{R}_{0}, \mathbf{r}_{n}, \mathbf{r}_{\mathrm{cm}}$ Distance & $\mathrm{m}$ \\
$m, m_{n}$ & Mass & $\mathrm{kg}$ \\
$L_{p}, L_{q}, L_{r}$ & Distance & $\mathrm{m}$ \\
$b_{r e f}, c_{r e f}$ & Distance & $\mathrm{m}$ \\
$S_{r e f}$ & Area & $\mathrm{m}$ \\
\hline
\end{tabular}




\section{References}

[1] Terrence A. Weisshaar. Morphing aircraft technology - new shapes for aircraft design. NATO Research and Technology Organisation (RTO), 2006.

[2] Rafic Ajaj, Christopher Beaverstock, and Michael Friswell. Morphing aircraft: The need for a new design philosophy. Aerospace Science and Technology, page 12, 2015.

[3] Charles H. Gibbs-Smith. Aviation: An Historical Survey from Its Origins to the End of World War, volume 1. NMSI Trading Ltd., 2 edition, 2013.

[4] Onur Bilgen Michael I. Friswell Daniel J. Inman Silvestro Barbarino, Rafic M. Ajaj. A review of morphing aircraft. Journal Of Intelligent Material Systems And Structures, page 12, 2011.

[5] Nicholas B. Cramer, Daniel W. Cellucci, Olivia B. Formoso, Christine E. Gregg, Benjamin E. Jenett, Joseph H. Kim, Martynas Lendraitis, Sean S. Swei, Greenfield T. Trinh, Khanh V Trinh, and Kenneth C. Cheung. Elastic shape morphing of ultralight structures by programmable assembly. Journal Of Smart Materials and Structures, page 14, 2019.

[6] Daochun Li, Shiwei Zhao, Andrea Da Ronch, Jinwu Xiang, Jernej Drofelnik, Yongchao Li, Lu Zhang, Yining Wu, Markus Kintscher, Hans Peter Monner, Anton Rudenko, Shijun Guo, Weilong Yin, Johannes Kirn, Stefan Storm, and Roeland De Breuker. A review of modelling and analysis of morphing wings. Progress in Aerospace Sciences, page 12, 2018.

[7] Alexander Pankonien and Daniel J. Inman. Experimental testing of spanwise morphing trailing edge concept. Active and Passive Structures and Integrated Systems, 2013.

[8] A. Tarabi, S. Ghasemloo, and M. Mani. Experimental investigation of a variable-span morphing wing model for an unmanned aerial vehicle. The Brazilian Society of Mechanical Sciences and Engineering, page 9, 2015.

[9] flexsys, 2018.

[10] Kenneth E. Boothe. Dynamic modeling and flight control of morphing air vehicles. Master of science, University of Florida, Gainesville, Florida, 2004.

[11] Thomas S. Koch. Stability and control of a morphing vehicle. Master of science in aeronautics and astronautics, University of Washington, Seattle, Washington, 2012.

[12] B. Obradovic and K. Subbarao. Modeling of flight dynamics of morphing-wing aircraft. Journal of aircraft, 48:11, 2011
[13] N. Ameri, M. H. Lowenberg, and M. I. Friswell. Modelling the dynamic response of a morphing wing with active winglets. Hilton Head, South Carolina, 2007. Atmospheric Flight Mechanics Conference and Exhibit.

[14] Joaquim R.R.A. Martins David A. Burdette. Design of a transonic wing with an adaptive morphing trailing edge via aerostructural optimization. Aerospace Science and Technology, page 12, 2018.

[15] Eun Jung Chae, Amin Moosavian, Alexander M. Pankonien, and Daniel J. Inman. A comparative study of a morphing wing. Conference on Smart Materials, Adaptive Structures and Intelligent Systems, 2017.

[16] Ivan Wang, S. Chad Gibbs, and Earl H. Dowell. Aeroelastic model of multisegmented folding wings: Theory and experiment. Journal of aircraft, 49:11, 2012.

[17] Thiemo M. Kier. Comparison of unsteady aerodynamic modelling methodologies with respect to flight loads analysis. AIAA Atmospheric Flight Mechanics Conference, 2005.

[18] Hildebrando F. de Castro, Pedro Paglione, and Carlos Henrique Ribeiro. Exponential mapping controller applied to aircraft. AIAA Guidance, Navigation, and Control Conference, 2012.

[19] Ahmed A. Shabana. Dynamics of Multibody Systems, volume 1. Cambridge University Press, 3 edition, 2005.

[20] T. Theodorsen and I. E. Garrick. Mechanism of flutter. a theoretical and experimental investigation of the flutter problem, 1940.

[21] Theodore Theodorsen. General theory of aerodynamic instability and the mechanism of flutter, 1949.

[22] J. G. Barmby, H. J. Cunningham, and I. E. Garrick. Study of effects of sweep on the flutter of cantilever wings, 1951.

[23] Jr. E. Carson Yates. Calculation of flutter characteristics for finite-span swept or unswept wings at subsonic and supersonic speeds by a modified strip analysis, 1958.

[24] Grégori Pogorzelski. Dinâmica de aeronaves flexíveis empregando teoria das faixas não-estacionária. Master of science, Instituto Tecnológico de Aeronáutica, São Jóse dos Campos, SP-Brasil, 2010.

[25] Raymond L. Bisplinghoff, Holt Ashley, and Robert L. Halfman. Aerolasticity. Dover Publications, Inc., 1996.

[26] K. Mohamed Hussain, R. Allwyn Rajendran Zepherin, M. Shantha Kumar, and S.M. Giriraj Kumar. Comparison of pid controller tuning methods with genetic algorithm for foptd system. Journal of Engineering Research and Applications, page 7, 2014.

[27] Henrik Grankvist. Autopilot design and path planning for a uav. Defence and Security, Systems and Technology, 2006. 